\title{
Influence of chromosome structure on the degree of meiotic pairing of intercultivar wheat hybrids (Triticum aestivum L.)
}

C. Vega*,

A. Fominaya $†$ and E. Ferrert
* Departamento de Genética, Facultad de Biología, Universidad Complutense, 28040, Spain.

$\dagger$ Departamento de Genética, Facultad de Ciencias, Universidad de Alcalá de Henares, Apdo, 20 Alcalá de Henares, Madrid, Spain.

The hybrid asynapsis in the F1 between 20 wheat cultivars and "Chinese Spring" was analysed using the $C$-banding technique. This allowed unequivocal identification of nine meiotic chromosomes $(4 \mathrm{~A}, 7 \mathrm{~A}$ and all seven of the $\mathrm{B}$ genome). The number of unbound chromosome arms shown by different chromosomes in different hybrids appeared to indicate the existence of a differential contribution to the asynapsis of each chromosome in every hybrid. The hybrid asynapsis was not only due to the chromosomes involved in large reciprocal translocations. Other causes are also suggested namely, cytologically cryptic or molecular polymorphisms and non allelic genetic effects.

\section{INTRODUCTION}

Chromosome pairing at metaphase I in intercultivar hybrids of wheat is less regular than their corresponding parents, resulting in a higher frequency of cells with univalents and a lesser number of chiasma per cell (Hollingshead, 1932; Morrison, 1953; Person, 1956; Riley and Kimber, 1961; Watanabe, 1962; Röbbelen, 1968; Schlegel and Mettin, 1981).

This fact has been explained in different ways. The existence of structural differences mainly translocations between wheat cultivars and their possible role in gene evolution have been given considerable attention following the work of Baker and McIntosh (1966) and other similar works (Vega and Lacadena, 1982, 1983; Fominaya and Jouve, 1985, 1986).

Structural heterozygosity which could appear in F1 between parental carriers of different chromosome arrangements can produce failure in pairing (Watanabe, 1962). Dvořák and McGuire (1981) considered that the reduced pairing between homologous heterozygous chromosomes (homologous of different cultivars) in relation to

† To whom all correspondence should be sent. that observed in the parental lines could be caused by polymorphisms in the nucleotide sequences (nonstructural chromosome variation) among wheat cultivars.

It has been argued that the optimum level of wheat pairing is related to the system of predominating crossing in favour of a genetic balance based on homozygosity; Person (1956) observed a gradual recovery of meiotic stability after backcrossing of the hybrid; Riley and Law (1965) concluded that such recovery of the stability was too gradual to accord with that expected if homozygosity alone was responsible for stable pairing. They considered that the reduced chromosome pairing occurring in wheat intercultivar hybrids was a manifestation of "negative heterosis" caused by gene interactions. Schlegel and Mettin (1981) established for a small number of genes, at least two major genes, which controlled the asynapsis level, and one of those was located by Mettin and Kimber (1983) on chromosome 3A.

In the present work we have studied the meiotic behaviour of 20 hybrids of wheat cultivars using "Chinese Spring" as the common parent; to determine whether or not differential contributions to the asynapsis of the chromosomes exist that could be identified by $C$-banding techniques. 


\section{MATERIALS AND METHODS}

Monosomic or disomic hybrids of "Chinese Spring" and the following 20 cultivars of hexaploid wheat were used: "Boulmiche", "Cabezorro", "Calatrava", "Canaleja", "Candeal de Teruel", "Caspino", "Cierzo", "Flambar II", "Hembrilla de Jaca", "J-I”, “Magdalena", "Matador", "Mentana", "Oroel", "San Bruno", "Sansa", "Saria", "Siete Cerros", "Talento", and "Toroma".

Chromosome numbers were determined in root tips by conventional Feulgen-staining techniques. Plants were grown in greenhouse conditions. Anthers for meiotic analysis were collected and fixed in alcohol-acetic acid, $3: 1$. The Giemsa procedure ( $C$-banding) was used for differential staining of chromosomes in meiosis as previously described (Jouve et al., 1980). More than 50 pollen mother cells (PMCs) were analysed per plant. Giemsa $C$-banding technique permitted unequivocal recognition of nine meiotic chromosomes (4A, 7A and the seven of the $B$ genome) at first metaphase (MI) as previously reported (Ferrer et al. 1984a). The $C$-banding pattern for these chromosomes was consistent with the one described by Gill and Kimber (1974), Seal (1982), Van Nieckerk and Pienaar (1983), and Endo and Gill (1984).

The degree of asynapsis is generally expressed by the frequency of PMCs at MI with at least a pair of univalents. We consider that the higher number of open bivalents observed in intervarietal hybrids compared with the cultivars is also an asynaptic effect.

The number of unbound chromosome arms at first metaphase was used instead of the frequency of univalents. In each hybrid we determined: (a) The total number of unbound arms, (b) the number of unbound arms of each identified chromosome and (c) the number of unbound arms in the whole of chromosomes which appear unbanded with the technique used.

In order to establish the presence or not of differences between hybrids in the degree of asynapsis as well as in the participation of chromosomes or groups of identified chromosomes, contingency chi-square $\left(\chi^{2}\right)$ test was calculated comparing the observed frequencies of unbound arms.

\section{RESULTS}

Most of the studied hybrids showed multivalent association, mainly quadrivalents indicating the existence of reciprocal translocations which differentiated the cultivars from "Chinese Spring".
In some cases the chromosomes and chromosome arms involved in the interchange have been identified by band pattern.

The frequency of PMCs with multivalents was closely related to the size of the translocated segment. These results are shown in table 1 .

We must highlight the following points:

(a) There are big differences in the size of the translocated segments;

(b) The chromosomes $5 B$ and $7 B$ are involved in one translocation ( $5 B S-7 B S / 5 B L-7 B L)$ which is carried by several cultivars (fig. 1). Such cultivars (Talento, Cierzo, Oroel, Canaleja and Boulmiche) have common ancestors in their phylogeny, therefore it can be assumed, that the translocation is transmitted rather than being of a recurrent origin. This translocation was assumed to be of great size owing to the high frequency of PMCs with quadrivalents ( 80 per cent) and this was proved after observing the mitotic chromosomes. This translocation alters the characteristic morphology of chromosomes $5 B$ and $7 B$ of "Chinese Spring". The chromosome observations at mitotic metaphase have shown changes in relation to "Chinese Spring" (fig. 2).

(c) Another large translocation appears in "Chinese Spring" $\times$ "Cabezorro" among chromosomes unidentified with the $C$-banding technique used.

(d) It has to be noted that those chromosomes involved in translocations are not the only ones that can appear as univalents; moreover, translocated chromosomes do not form univalents with the highest frequency (table 1 and fig. 3).

If the structural chromosome differentiation caused the asynapsis, the chromosomes involved in the translocations would be the ones with the higher number of unbound arms. On the other hand if non-structural chromosome differentiation were the cause, a greater number of chromosomes would participate in asynapsis and, it would be expected that the involved chromosomes would be different for each hybrid.

The frequency of unbound arms in the analysed hybrids which ranged from $7 \cdot 69$ per cent to $13 \cdot 18$ per cent are graphically shown in fig. 4 . We have compared three groups of hybrids $G 1, G 2$ and $G 3$ with differing total unbound arm frequencies.

In each group there was a differential participation of the chromosomes belonging to each genome. The rates between the total number of unbound arms of the chromosomes of $B$ genome as a whole and the total number of unbound arms were significantly different within these groups. In consequence, subgroups of hybrids which showed 
Table 1 Analysis of chromosome pairing in intercultivar wheat hybrids (Triticum aestivum L.)

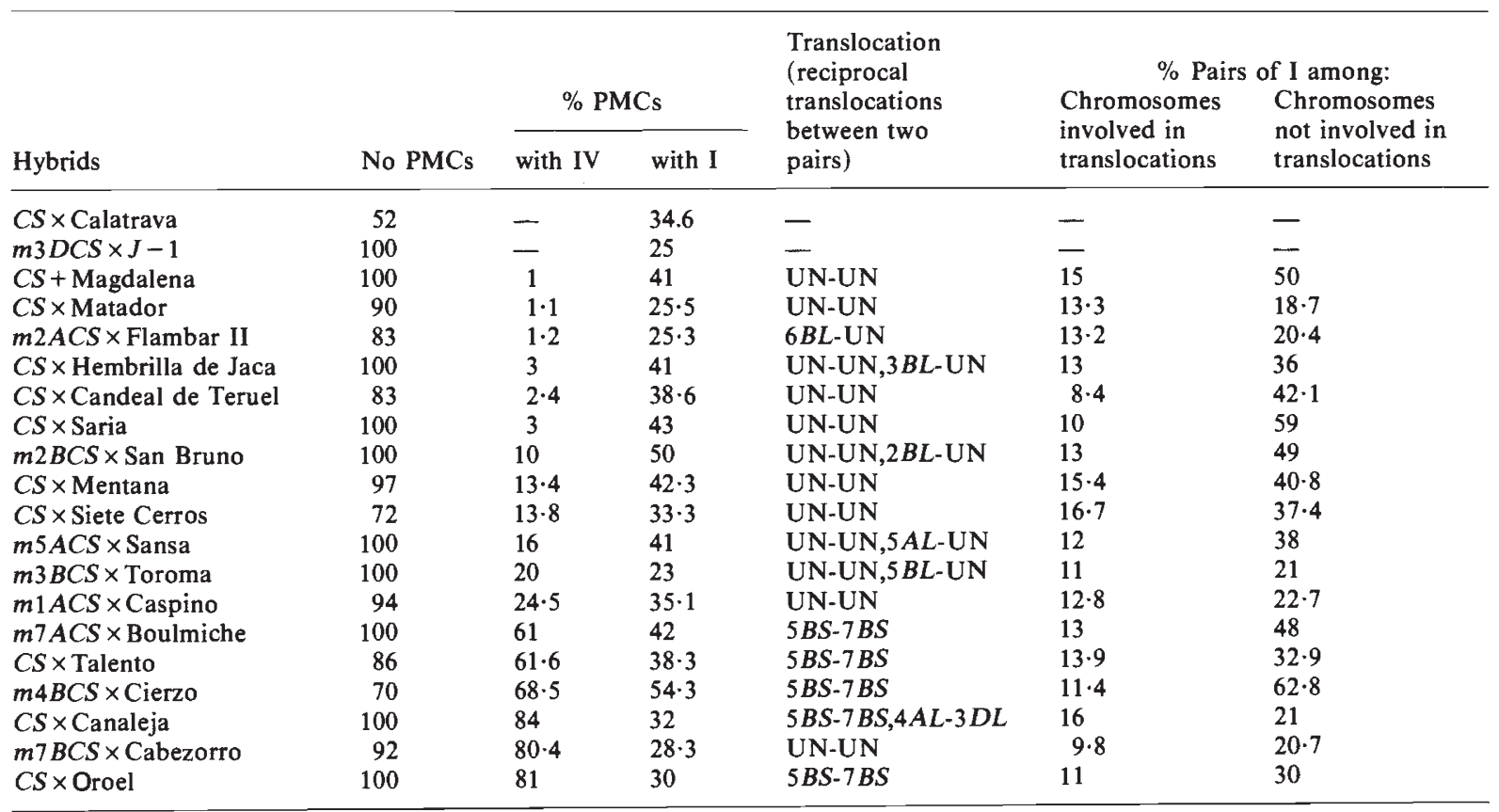

a homogeneous behaviour in the degree of asynapsis of the chromosomes of the $B$ genome were established. Such subgroups were named $G 1-1$, $G 1-2, G 2-1, G 2-2, G 2-3$; if all the wheat chromosomes were to have an equal participation in the asynapsis, the $B$ genome would have 33 per cent of the total unbound arms. However, as can be seen in fig. 4 the minimum value found for $G 1-1$ was 44.05 per cent. The participation of each identified chromosome of the $B$ genome $(1 B, 2 B \ldots 7 B)$ in every subgroup has been compared with the

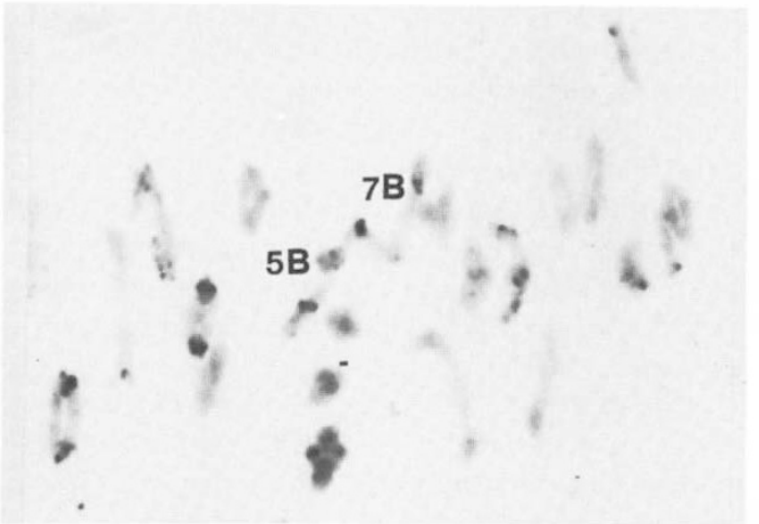

Figure 1 Photomicrograph of PMC at first metaphase stained by Giemsa in a reciprocal translocation heterozygote involving common wheat chromosomes $5 B$ and $7 B$. number of total unbound arms of that genome. Significant differences were observed between the groups and chromosomes as can be seen in table 2 . If each $B$ genome chromosome were to participate in a similar way in the asynapsis, one would expect a 14 per cent of unbound arms for each chromosome. However, the differences on the asynapsis for each identified chromosome in the different hybrids can be observed (table 2).

For the $A$ and $D$ genome chromosomes we must note:

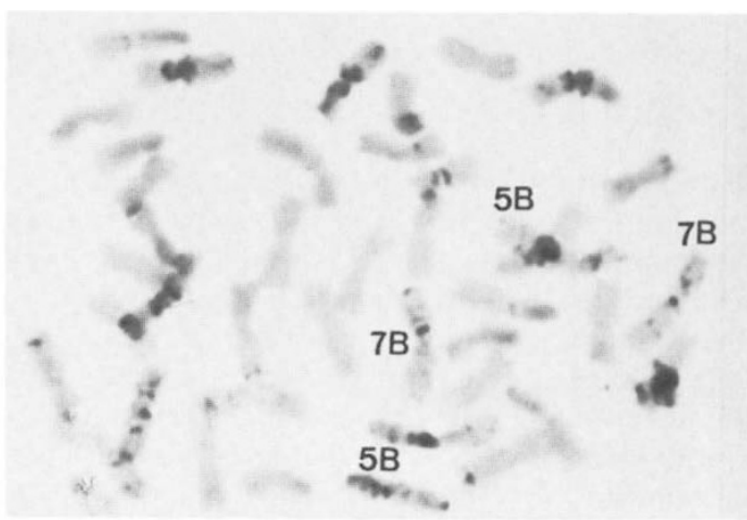

Figure 2 C-banded mitotic metaphase cnromosomes or tne "Boulmiche" common wheat, showing different morphology in the $5 B$ and $7 B$ chromosomes compared with "Chinese Spring". 


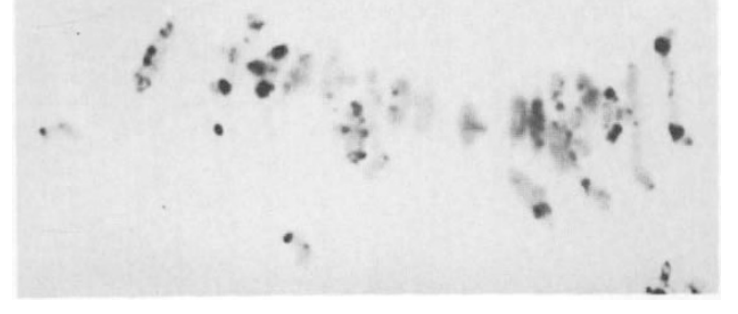

Figure 3 First metaphase plate with 15 ring bivalents +5 open bivalents $(4 A, 7 A, 5 B, 7 B, U N)+2$ univalents $(4 B)$.

(a) there is a great difference in the behaviour of the two identified chromosomes belonging to the $A$ genome; the $4 A$ chromosome makes a high contribution to the asynapsis frequency in both genomes while the $7 \mathrm{~A}$ chromosome has a very low value.

(b) In a similar way to the $B$ genome, there is a great difference in the hybrids.

(c) Although the mean contribution of the unidentified chromosomes to the asynapsis has similar values in the hybrids ( 7 per cent) the existence of differences in this contribution is possible, such as occurs in the cases of $4 A, 7 A$ and the $B$ genome chromosomes. Chromosomes $5 B$ and $7 B$ have a principal role in the asynapsis of the hybrids with reciprocal translocations among them, but this does not mean that they are the ones with the higher number of unbound arms. On the other hand, the other hybrids without the reciprocal translocation $5 B / 7 B$ ("Chinese Spring" $\times$ "Saria", "Chinese Spring" $\times$ "Matador"...) have a high frequency of unbound arms for those chromosomes.

\section{DISCUSSION}

Pairing instability after intervarietal hybridisation of homozygous cultivars is a well known phenomenon. Schlegel and Mettin (1981) analysed 64 intervarietal hybrids using "Chinese Spring" as the common parent and observed the existence of different levels of asynapsis which appeared stable in the following years. Most of the hybrids showed frequencies of cells with 20 per cent univalents. In our case the frequencies were higher (35-40 per cent) and could perhaps be attributed to different environmental conditions.

Riley and Kimber (1961) assumed a proportional participation in the asynapsis by the chromosomes belonging to different genomes. Our results were not in accord with that; $B$ genome and $4 A$ chromosomes play a more important part than expected in asynapsis. Miller and Reader (1985) have found significant differences, analysing chiasma frequencies among isolated bivalents of wheat. They observed that some homologous bivalents, such as $5 \mathrm{~B}$ chromosome, frequently formed only a rod bivalent moreover, also they found a highly significant low chiasma frequencies for the $4 A$ chromosomes. Our results agree with these authors because the contribution of both chromosomes to the asynapsis was important. Factors such as length, DNA content and the amount of heterochromatin, are indicated as some of the causes that could explain the differential pairing of the chromosomes belonging to different wheat genomes (Dvořák and McGuire, 1981; Ferrer et al., 1984b).

The differential contribution of each identified chromosome to the asynapsis of each hybrid seems to indicate that in addition to the factors that establish differences between genomes others exist that individually act on the chromosomes of the different cultivars.

The structural chromosome differentiation indicated by the appearance of multivalent configurations at $M I$ in $F 1$ hybrids is not the sole or principal cause. Watanabe (1962) postulated the existence of structural differences in the parental karyotypes, such as rearrangements or deficiencies too small to be cytologically detected. If this were so, they should be spread over many chromosomes and should occur often in order to explain the differences between cultivars with close genealogies.

One additional explanation was proposed by Dvořák and McGuire (1981) and Crossway and Dvořák (1984) who considered that differentiation between wheat cultivars was due mainly to chromosome changes at the nucleotide sequence level. Dvořák and Appels (1982) assumed that nonstructural variations would mainly affect the non-codifying regions of the chromosomes which are not evenly distributed in the genome. With both hypotheses chromosomal heterozygosity would be mainly responsible for the hybrid asynapsis.

The existence of a genetic control of asynapsis has been pointed out by Riley and Law (1965), Schlegel and Mettin (1981) and Mettin and Kimber (1983). The reduced meiotic pairing observed in intervarietal hybrids would be due to the genotype, since all the cultivars are assumed to be homozygous, and the differences in asynapsis levels 
Table 2 Analysis of the contribution to the asynapsis of every identified chromosome by $C$-banding and the group of unidentified chromosomes in intercultivar hybrids of common wheat

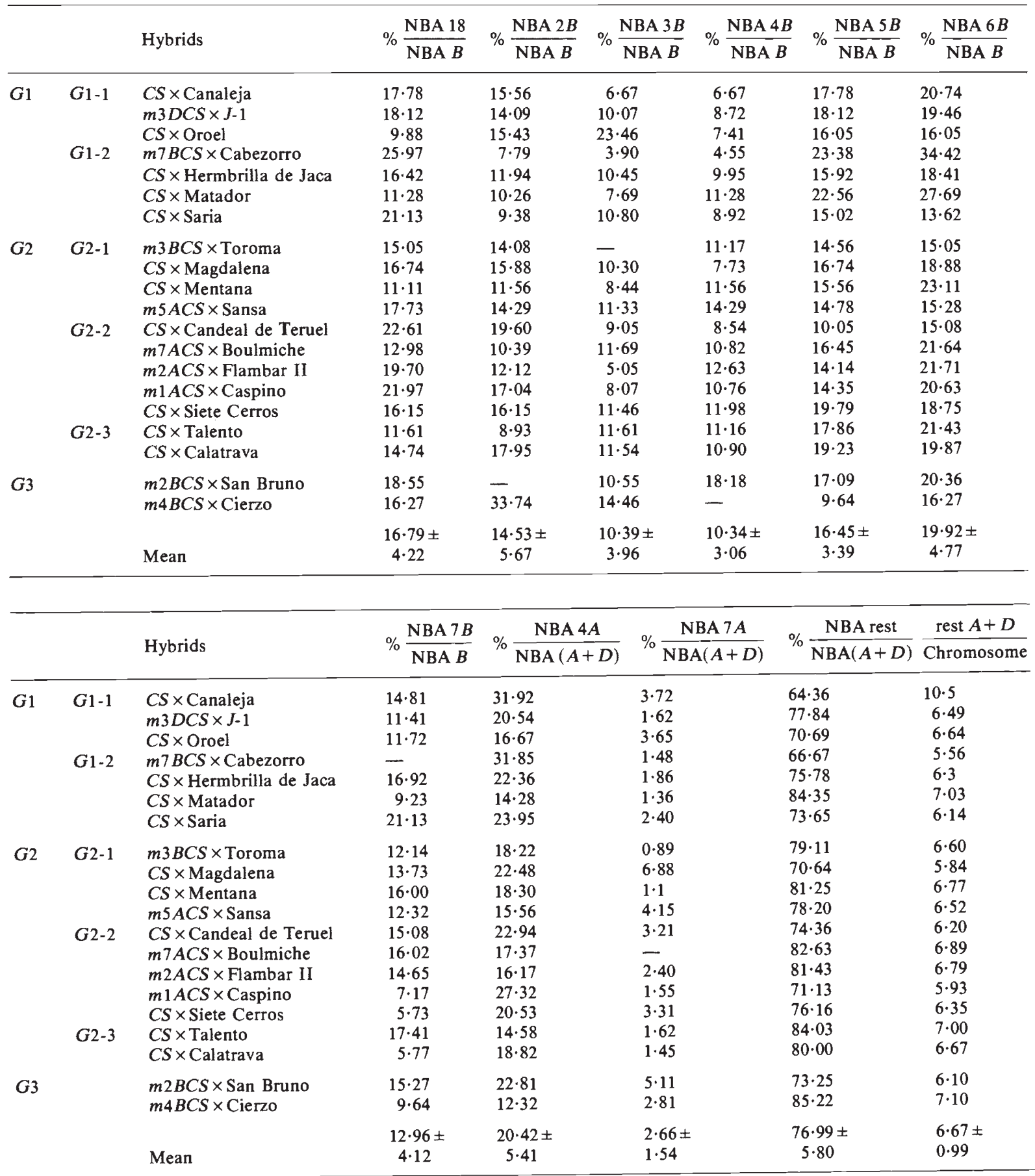

Note: NBA indicates unbound arms 


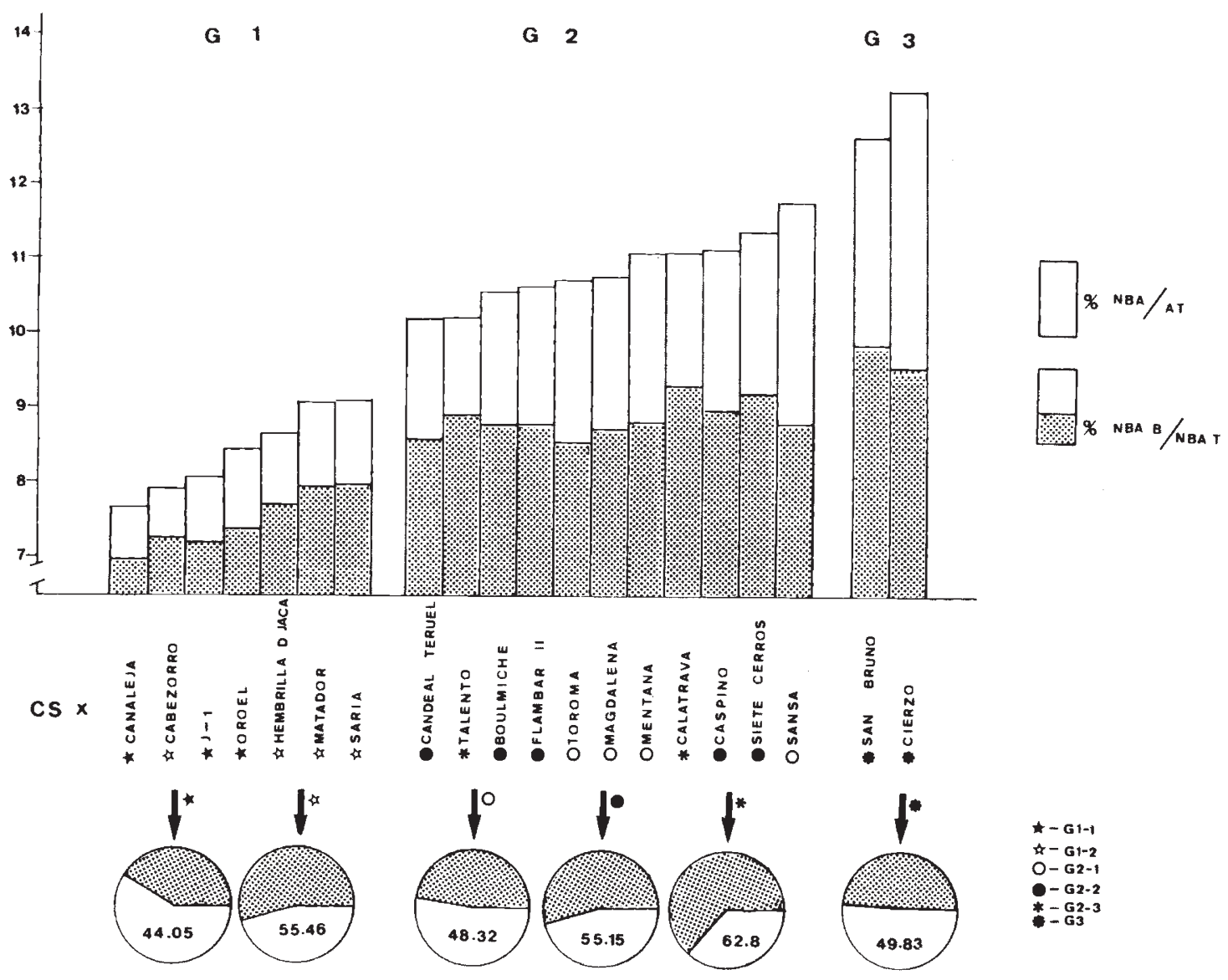

Figure 4 Frequency histogram of number of unbound arms/total arms $(N B A / A T)$ for the different hybrids analysed. The cyclograms show the mean contribution of the $B$ genome to the asynapsis in the different subgroups.

observed in the hybrids must be due more to the new genic combinations in the hybrids than to heterozygosity. If this were so, the preferential asynapsis observed that acts on different chromosome pairs in the different hybrids would indicate a differential effect on each chromosome of the regulating genetic systems of meiotic pairing in wheat. It is possible that the differential genetic activity on each chromosome is executed through nucleotide sequences, involved in the non-structural chromosomal polymorphism, that could avoid a proper synapsis or could interfere with the occurrence of chiasmata.

\section{REFERENCES}

BAKER, E. AND MCINTOSH, R. A. 1966. Chromosome translocations identified in varieties of common wheat. Can.J. Genet. Cytol, 8, 592-599.
CROSSWAY, A. AND DVOŔÁK, J. 1984. Distribution of nonstructural variation along three chromosome arms between wheat cultivars Chinese Spring and Cheyenne. Genetics, 106, 309-324.

DVOŘÁK, J. AND MCGUIRE, P. 1981. Nonstructural chromosome differentiations among wheat cultivars, with special reference to differentiation of chromosomes in related species. Genetics, 97, 391-414.

DVOŘÁK, J. AND APPELS, R. 1982. Chromosome and nucleotide sequence differentiation in genomes of polyploid Triticum species. Theor. Appl. Genet. 63, 349-360.

ENDO, T. R. AND GILL, B. S. 1984. Somatic karyotype, heterochromatin distribution, and nature of chromosome differentiation in common wheat, Triticum aestivum L. em Thell. Chromosoma, 89, 361-369.

FERRER, E., GONZALEZ, J. M. AND JOUVE, N. 1984a. Identification of $C$-banded chromosomes in meiosis of common wheat, Triticum aestivum L. Theor. Appl. Genet., 67, 257261.

FERRER, E., GONZALEZ, J. M. AND JOUVE, N. 1984b. The meiotic pairing of nine wheat chromosomes. Theor. Appl. Genet., 69, 193-198.

FOMINAYA, A. AND JOUVE, N. 1985. $C$-banding at meiosis as a means of analyzing cytogenetic structure in wheat. Can. J. Genet. Cytol., 27, 689-696. 
FOMINAYA, A. AND JOUVE, N. 1986. Analysis of interference in a double interchange heterozygote of wheat (Triticum aestivum L.). Heredity, 56, 1-6.

GILL, B. S. AND KIMBER, G. 1974. Giemsa $C$-banding and the evolution of wheat. Proc. Nat. Acad. Sci., 71, 4086-4090.

HOLLINGSHEAD, L. 1932. The occurrence of unpaired chromosomes in hybrids between varieties of Triticum vulgare. Cytologia, 3, 119-141.

JOUVE, N., DIEZ, N. AND RODRIGUEZ, M. 1980. $C$-banding in $6 x$-triticale $\times$ Secale cereale L. Hybrid cytogenetics. Theor. Appl. Genet., 57, 75-79.

METTIN, D. AND KIMBER, G. 1983. The monosomic analysis of the genes for 'hybrid desynapsis' in common wheat. Proc. 6th. Int. Wheat Genet. Symp. Kyoto, pp. 315-319.

MILLER, T. E. AND READER, S. M. 1985. The effect of increased dosage of wheat chromosomes on chromosome pairing and an analysis of the chiasma frequencies of individual wheat bivalents. Can. J. Genet. Cytol., 27, 421-425.

MORRISON, J. W. 1953. Chromosome behaviour in wheat monosomics Heredity, 7, 203-217.

NIEKER, H. A. VAN. AND PIENAAR, R. V. 1983. Morphology and linear $C$-band differentiation of Triticum aestivum L. em Thell $v$. aestivum cv. 'Chinese Spring' chromosomes. Cereal. Res. Commun., 11, 115-122.
PERSON, C. 1956. Some aspects of monosomic wheat breeding. Can. J. Bot., 34, 60-70.

RILEY, R. AND KIMBER, G, 1961. Aneuploids and the cytogenetic structure of wheat varietal populations. Heredity, 16, 275-290.

RILEY, R. AND LAW, C. N., 1965. Genetic variation in chromosome pairing. Adv. Genet., 13, 57-114.

RöbBELEN, G. 1968. Desynapsis als Fehlerquelle bei der Aufstellung von Monosomen- Sortimenten des Weizens. $Z$. Pflanzenzuchtg, 60, 1-18.

SCHLEGEL, G. AND METTIN, D. 1981. A contribution to the genetics of 'Hybrid desynapsis' in common wheat, T. aestivum L. Biol. Zbl, 100, 395-406.

SEAL, A. G. 1982. $C$-banded wheat chromosomes in wheat and triticale. Theor. Appl. Genet., 63, 49-56.

VEGA, C. AND LACADENA, J. R. 1982. Cytogenetic structure of common wheat cultivars from or introduced into Spain. Theor. Appl. Genet., 61, 123-129.

VEGA, C. AND LACADENA J. R. 1983. Identification of two chromosomal interchanges in $\mathrm{cv}$. Canaleja of common wheat, Triticum aestivum L. Euphytica, 32, 485-491.

WATANABE, Y. 1962. Meiotic irregularities in intervarietal hybrids of common wheat. Whear Infor. Serv., 14, 5-7. 